\title{
Diurnal Salivary Cortisol Profiles in Patients with Cushing's Syndrome
}

\author{
Authors

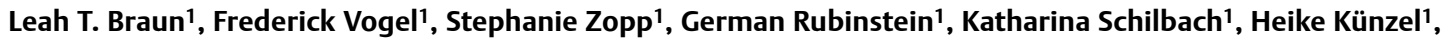 \\ Felix Beuschlein ${ }^{1,2}$, Martin Reincke ${ }^{1}$
}

\section{Affiliations}

1 Medizinische Klinik und Poliklinik IV, LMU Klinikum, Ludwig-Maximilians-Universität München, Munich, Germany

2 Klinik für Endokrinologie, Diabetologie und Klinische Ernährung, Universitätsspital Zürich, Switzerland

\section{Key words}

hypercortisolism, Cushing's disease, circadian rhythm, adrenocorticotropin, pituitary, adrenal

received $\quad 05.10 .2021$

revised 25.11.2021

accepted 07.12 .2021

published online 17.01 .2022

Bibliography

Exp Clin Endocrinol Diabetes 2022; 130: 434-438

DOI 10.1055/a-1719-5381

ISSN 0947-7349

(c) 2022. Thieme. All rights reserved.

Georg Thieme Verlag KG, Rüdigerstraße 14,

70469 Stuttgart, Germany

\section{Correspondence}

Dr. Leah Braun

Medizinische Klinik und Poliklinik IV

LMU Klinikum

Ludwig-Maximilians-Universität München

Munich Germany

Ziemssenstraße 5

80336 München

Germany

Tel: + 49/89/440057 334, Fax: +49/89/4400573339

Leah.Braun@med.uni-muenchen.de

\section{ABSTRACT}

Background Quantification of salivary cortisol is one of the highly sensitive and specific screening parameters for Cushing's syndrome (CS). However, only late-night salivary cortisol is part of the standard screening procedure. In this study, we aimed to analyze salivary cortisol day profiles in patients with different types of CS to test whether specific patterns might be relevant for diagnosis and subtyping.

Material and Methods Among 428 patients including those with confirmed Cushing's syndrome $(\mathrm{N}=111$, of those 75 with Cushing's disease, 27 patients with adrenal CS and nine patients with ectopic $C S)$, autonomous cortisol secretion $(N=39)$ or exclusion of CS (control group, $\mathrm{N}=278$ ) salivary cortisol was measured five times a day.

Results At each of the five time points, salivary cortisol was significantly higher in patients with CS compared to the control group $(p \leq 0.001)$. Using the entire profile instead of one single salivary cortisol at 11 p.m. improved diagnostic accuracy (85 vs. $91 \%$ ) slightly. Patients with ACTH-dependent CS had higher salivary cortisol levels than patients with adrenal CS. Also, morning cortisol was significantly higher in patients with ectopic CS than in patients with Cushing's disease $(p=0.04)$. Nevertheless, there was a strong overlap between diurnal profiles, and the diagnostic yield for subtyping was low.

Discussion The study results show that using diurnal salivary cortisol profiles for CS diagnosis results in a limited increase in diagnostic accuracy. With significant differences between Cushing subtypes, cortisol profiles are not useful in everyday clinical practice for subtyping of CS.

\section{Introduction}

Endogenous hypercortisolism, also referred to as Cushing's syndrome (CS) is a rare but severe disease affecting approximately 0.2-5.0 per million people annually [1]. The diagnosis of CS can be challenging due to the variety of clinical signs and symptoms. Standard screening tests for CS include the $1 \mathrm{mg}$ low dose dexamethasone suppression test (LDDST), late-night salivary cortisol, and the cortisol levels in a 24-hour urine sample (urine free cortisol, UFC) [2]. All tests are quite sensitive and specific [2, 3]. However, overall test accuracy is dependent on assays and pre-analytic conditions. Also, due to the rareness of the disease, pre-test probability is low, and therefore, despite high sensitivity and specificity, there can be a high rate of false-positive and even false-negative results [4]. In the past decades, new diagnostic methods such as 
plasma steroid profiling, face recognition, and clinical scores have been developed to increase the accuracy of diagnosis and to differentiate subtypes [4]. However, none of the mentioned methods has yet been established in clinical routine.

Of the standard screening tests, late-night salivary cortisol seems to be the best test for early detection of the recurrence of Cushing's disease [5,6]. It can be performed easily on an outpatient basis. There causes for false negative or positive results are only a few, such as smoking, gum bleeding, contamination with steroidcontaining ointments, or disturbed cortisol rhythms due to jet lag or shift working [4]. However, there is a lack of knowledge about the use of the daily salivary cortisol profile in diagnosing and subtyping CS. In a study including 100 healthy subjects (both children and adults) and 150 patients with CS, cut-offs for saliva cortisol at different points of time were established but salivary profiles in different subtypes were not analyzed [7]. In their study, Castro et al. observed that salivary cortisol in patients with CS (33 subjects) was elevated at 9:00 a.m., 5 p.m. and 11 p.m. Sensitivity can be improved by combining the $1 \mathrm{mg}$ low dose dexamethasone suppression test and measuring salivary cortisol [8]. Similar results were observed by Laudat et al. [9].

From a pathophysiological perspective, it is known that patients having CS suffer from a disturbed diurnal cortisol rhythm. This would make salivary cortisol measurements an ideal method for subtype diagnosis between the pituitary, adrenal, and ectopic forms. Although late-night salivary cortisol measurement is a firmly established screening tool, no study has systematically analyzed the value of a five-point diurnal profile in diagnosing CS and identifying Cushing subtypes. Based on this background, the current study aimed to investigate daily salivary cortisol profiles in patients with ACTH-dependent and ACTH-independent CS.

\section{Material and Methods}

This study was part of the prospective German Cushing's registry founded in 2012. Since then, 520 patients with suspected or confirmed CS were prospectively included in the registry. The structure of the registry has been described previously $[10,11]$. Ethical approval was obtained (195-09) and all patients gave written informed consent.

All patients underwent a standardized clinical examination and a standardized biochemical screening including the $1 \mathrm{mg}$-lowdose-dexamethasone-suppression-test, collection of 24-hours urine (for UFC), and a salivary cortisol day-profile. For the latter, patients were asked to collect saliva at 8:00 and 12:00 a.m. and 4:00, $8: 00$, and 11:00 p.m. Patients were carefully instructed for the saliva collection. The laboratory analyses were performed at the Endocrine Laboratory of the Medizinische Klinik und Poliklinik IV, LMU Klinikum. Salivary cortisol was measured using an automated chemiluminescence immunoassay (CLIA, IDS-iSYS, Immunodiagnostic Systems, Boldon). Reference intervals were only established for late-night salivary cortisol (normal: $<1.5 \mathrm{ng} / \mathrm{mL}$, grey zone: $1.5-$ $2.2 \mathrm{ng} / \mathrm{mL}$ ); reference intervals for the other time points have not been established so far. After confirmation of CS, further subtyping including $\mathrm{CRH}$-Test, the $8 \mathrm{mg}$-high-dose-dexamethasone-suppression-test, imaging (sellar magnetic resonance imaging (MRI) or abdominal computed tomography (CT)) and - if needed - inferior petrosal sinus sampling was conducted.

Final diagnosis and subtypes of CS were based on unambiguous surgical confirmation of the underlying histopathological entity. The exclusion of CS was based on normal screening tests and clinical observation, as described earlier [12]. The definition of autonomous cortisol secretion followed the European Society of Endocrinology guideline definition [13].

Finally, CS was confirmed in 111 patients and excluded in 290 patients. In 39 patients with an adrenal incidentaloma, the final diagnosis was autonomous cortisol secretion. In the remaining patients, either other endocrine diseases, such as primary hyperaldosteronism, were diagnosed or patients were lost-to-follow-up before diagnosis was confirmed or excluded. In patients with the exclusion of CS, primary symptoms varied, such as obesity, hypertension, or hirsutism. Patients with cyclic CS were also excluded from the cohort $(\mathrm{N}=3)$. Of the prospectively observed patients with a confirmed exclusion or confirmation of CS, salivary day profiles were available for 428 patients. These patients formed the final study cohort.

\section{Statistical analysis}

For statistical analysis, SPSS 26 (IBM) was used. Results of standard biochemical screening including UFC, LDDST, serum cortisol, and ACTH are shown as median and ranges. As data did not show normal distribution, differences between groups were tested by nonparametric tests (Mann-Whitney $U$ test and Kruskal-Wallis test). Pvalues $<0.05$ were considered of statistical significance. Discriminant analysis with stepwise variable selection (saliva cortisol at different time points) was used to test which combination of saliva cortisol helps to discriminate the subtypes. The results from these analyses were compared with those of single late-night salivary cortisol determination.

\section{Results}

\section{Patient characteristics}

The biochemical characteristics of the patients are shown in $\triangleright$ Table 1 with $68 \%$ of patients having pituitary CS, $24 \%$ adrenal, and $8 \%$ were diagnosed with ectopic CS.

\section{Salivary profiles in patients with or without Cushing's syndrome}

Mean salivary cortisol concentrations were significantly higher in patients with CS at all five time points compared to patients in whom CS had been excluded ( $\vee$ Table 2 ). Overall, ranges were greater in patients with CS than in the control group ( $\triangleright$ Fig. 1). The use of the entire profile improved the diagnostic accuracy slightly compared to a single late-night salivary cortisol (85\% of cases correctly classified using only late-night salivary cortisol vs. $91 \%$ correctly classified using the entire profile). If additionally, the difference between morning and evening salivary cortisol was considered, accuracy improved further ( $92 \%$ correctly classified). Combining salivary cortisol profiles with UFC and low-dose-dexamethasone-suppression-test did not improve accuracy further 
- Table 1 Biochemical characteristics of the patients included in the study. (CS: Cushing's syndrome, LDDST = 1mg-dexamethasone-suppression-test. UFC: urinary free cortisol).

\begin{tabular}{|l|l|l|l|l|}
\hline & CS confirmed (N=111) & $\begin{array}{l}\text { Autonomous cortisol } \\
\text { secretion (N=39) }\end{array}$ & $\begin{array}{l}\text { CS excluded } \\
\text { (N=278) }\end{array}$ & $\begin{array}{l}\mathbf{p} \text {-values (all } \\
\text { groups) }\end{array}$ \\
\hline Sex & $74 \%$ females & $80 \%$ females & $77 \%$ females & - \\
\hline Age (years) & $46( \pm) 14$ & $63( \pm) 8$ & $39( \pm) 17$ & $\mathbf{p} \leq \mathbf{0 . 0 0 1}$ \\
\hline LDDST $(\mu \mathrm{g} / \mathrm{dL})$, Normal: $<1.8 \mu \mathrm{g} / \mathrm{dL}$ & $13.9(7.1-22.4)$ & $3.0(2.2-5.5)$ & $1.0(0.8-1.3)$ & $\mathbf{p} \leq \mathbf{0 . 0 0 1}$ \\
\hline UFC $(\mu \mathrm{g} / 24 \mathrm{~h})$, Normal: $<150 \mu \mathrm{g} / 24 \mathrm{~h}$ & $439(233-787)$ & $157(90-231)$ & $114(77-188)$ & $\mathbf{p} \leq \mathbf{0 . 0 0 1}$ \\
\hline ACTH $(\mathrm{pg} / \mathrm{mL})$, Normal: $4-50 \mathrm{pg} / \mathrm{mL}$ & $\begin{array}{l}44(10-77) \\
- \text { pituitary: } 59(36-83) \\
- \text { ectopic: } 104(52-221) \\
- \text { adrenal: } 3(2-5)\end{array}$ & $6.0(3.0-9.0)$ & $13(9-19)$ & $\mathbf{p} \leq \mathbf{0 . 0 0 1}$ \\
\hline $\begin{array}{l}\text { Morning Serum Cortisol }(\mu \mathrm{g} / \mathrm{dL}), \text { Normal: } \\
1.8-24 \mu \mathrm{g} / \mathrm{dL}\end{array}$ & $21.8(15.6-27.5)$ & $8.9(6.4-12.3)$ & $8.9(6.6-12.9)$ & $\mathbf{p} \leq \mathbf{0 . 0 0 1}$ \\
\hline
\end{tabular}

- Table 2 Daily salivary profiles of patients with or without Cushing's syndrome (CS).

\begin{tabular}{|c|c|c|c|c|c|}
\hline & 8:00 a.m. & 12:00 a.m. & 4:00 p.m. & 8:00 p.m. & 11:00 p.m. \\
\hline CS confirmed $(N=111)$ & $10.3(5.2-18.4)$ & $9.2(5.1-15.9)$ & $8.6(5.0-13.9)$ & $8.7(5.0-13.6)$ & $7.6(4.1-11.8)$ \\
\hline CS excluded $(N=278)$ & $6.5(4.4-9.7)$ & $3.0(2.0-5.0)$ & $2.4(1.7-3.4)$ & $1.3(0.9-2.1)$ & $1.0(0.7-1.7)$ \\
\hline Autonomous cortisol secretion $(\mathrm{N}=39$ ) & $7.6(4.2-10.7)$ & $3.6(2.6-6.0)$ & $3.2(2.2-5.0)$ & $2.4(1.7-4.0)$ & $2.6(1.6-3.6)$ \\
\hline p-values (all groups) & $\mathrm{p} \leq 0.001$ & $\mathrm{p} \leq 0.001$ & $\mathrm{p} \leq 0.001$ & $p \leq 0.001$ & $p \leq 0.001$ \\
\hline CS vs. CS Excluded & $p \leq 0.001$ & $p \leq 0.001$ & $\mathrm{p} \leq 0.001$ & $p \leq 0.001$ & $p \leq 0.001$ \\
\hline CS vs. Autonomous cortisol secretion & $p=0.04$ & $p \leq 0.001$ & $p \leq 0.001$ & $p \leq 0.001$ & $p=0.001$ \\
\hline Excluded vs. Autonomous & $\mathrm{p}=1.0$ & $\mathrm{p}=0.2$ & $p=0.01$ & $p \leq 0.001$ & $\mathrm{p} \leq 0.001$ \\
\hline
\end{tabular}
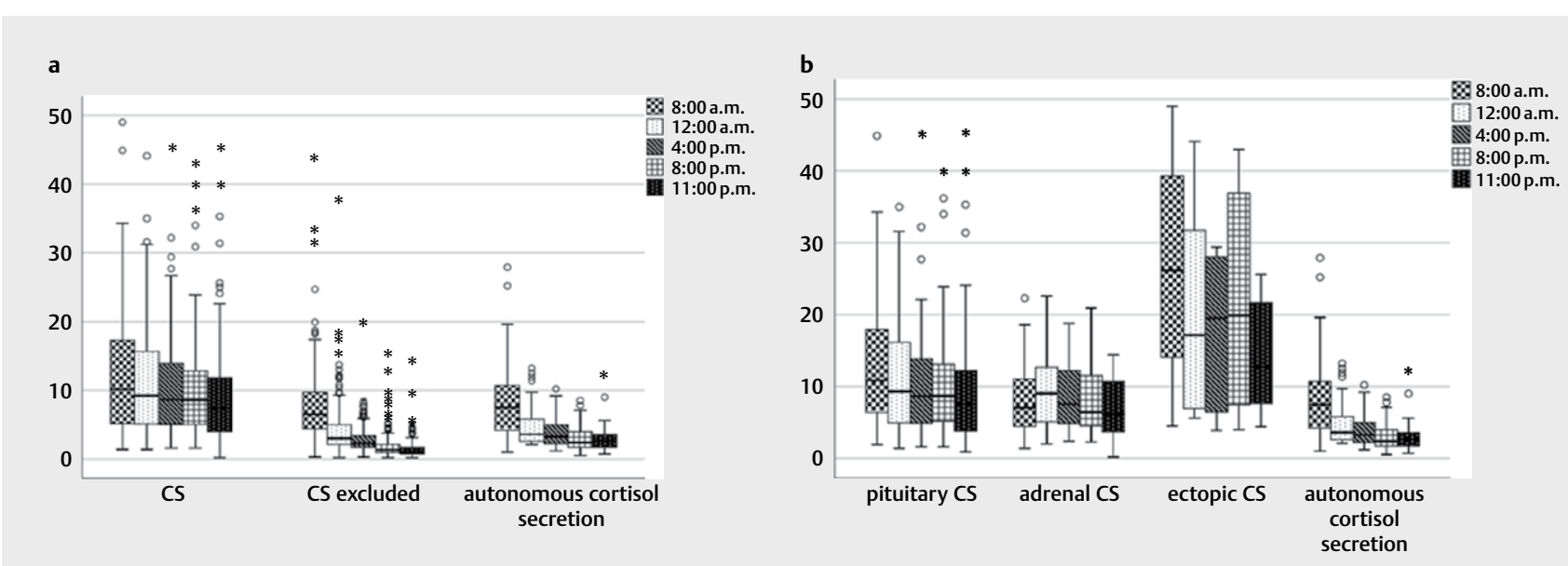

Fig. 1 a and b: Cortisol day profiles in all subgroups. CS=Cushing's syndrome. ${ }^{\circ *}=$ statistical outliers.

( $92 \%$ correctly classified) and was only slightly better than the combination of all three screening tests ( $91 \%$ correctly classified).

On average, late-night salivary cortisol levels were $85 \%$ (pituitary CS $83 \%$, adrenal CS $97 \%$, ectopic CS $61 \%$ ) of the morning cortisol. Subjects in whom CS was excluded, had low relative late-night cortisol levels (26\% of the 8:00 a.m. salivary cortisol concentration); whereas patients with autonomous cortisol secretion had significantly lower salivary cortisol levels compared to patients with CS at all time points but salivary cortisol in the afternoon and evening was significantly higher than in subjects with the exclusion of CS
( $p \leq 0.001)$, and relative late-night salivary cortisol levels were $51 \%$ of the morning levels.

\section{Salivary profiles in the different subtypes}

Salivary cortisol was higher in patients with pituitary CS and ectopic CS compared to those with adrenal CS. The difference between patients with ectopic and adrenal CS was significant in the morning (pituitary vs. adrenal CS) and at all time points except for noon.

Patients with pituitary and adrenal disease typically had constantly high cortisol levels during the entire day. In patients with 
- Table 3 Daily salivary profiles in patients with different subtypes of Cushing's syndrome (CS), Cushing's disease (CD).

\begin{tabular}{|l|l|l|l|l|l|}
\hline & $\mathbf{8 : 0 0}$ a.m. & $\mathbf{1 2 : 0 0}$ a.m. & $\mathbf{4 : 0 0} \mathbf{p . m .}$ & $\mathbf{8 : 0 0} \mathbf{p . m}$. & $\mathbf{1 1 : 0 0} \mathbf{p . m .}$ \\
\hline Pituitary CS (N= 75) & $10.5(5.2-18.8)$ & $9.3(4.9-16.5)$ & $8.6(4.8-13.8)$ & $8.8(5.1-14.3)$ & $7.9(4.1-11.9)$ \\
\hline Adrenal CS (N= 27) & $7.1(4.3-11.4)$ & $9.0(4.8-12.7)$ & $7.5(4.4-12.1)$ & $6.5(4.2-11.6)$ & $6.1(3.2-10.3)$ \\
\hline Ectopic CS (N=9) & $24.8(14.1-39.4)$ & $17.2(6.5-37.9)$ & $19.5(6.5-37.9)$ & $20.0(5.8-40.0)$ & $15.4(7.7-22.1)$ \\
\hline $\mathbf{p}$-values (All groups) & $\mathbf{p}=\mathbf{0 . 0 0 1}$ & $\mathrm{p}=0.1$ & $\mathbf{p}=\mathbf{0 . 0 4}$ & $\mathbf{p}=\mathbf{0 . 0 5}$ & $\mathbf{p}=\mathbf{0 . 0 3}$ \\
\hline $\mathbf{p}$-values (CD vs. Adrenal) & $\mathbf{p}=\mathbf{0 . 0 4}$ & - & $\mathrm{p}=1.0$ & $\mathrm{p}=0.5$ & $\mathrm{p}=0.4$ \\
\hline $\mathbf{p}$-values (CD vs. Ectopic) & $\mathbf{p}=\mathbf{0 . 0 4}$ & - & $\mathrm{p}=0.07$ & $\mathrm{p}=0.2$ & $\mathrm{p}=0.1$ \\
\hline $\begin{array}{l}\mathbf{p} \text {-values (Adrenal vs. } \\
\text { Ectopic) }\end{array}$ & $\mathbf{p}=\mathbf{0 . 0 0 1}$ & - & $\mathbf{p}=\mathbf{0 . 0 4}$ & $\mathbf{p}=\mathbf{0 . 0 4}$ & $\mathbf{p}=\mathbf{0 . 0 2}$ \\
\hline
\end{tabular}

ectopic CS, cortisol levels were especially high without any circadian rhythm; compared to patients with pituitary CS, salivary cortisol was significantly higher in the morning ( $>$ Table 3 ). Using the entire salivary profile or combining it with the other screening tests did not help to classify the subtypes correctly (data not shown).

\section{Discussion and conclusions}

Interpretation of screening results for the detection or exclusion of endogenous hypercortisolism can be challenging as elevated cortisol levels can be physiological in some cases $[2,14]$. Likewise, the lack of specificity of clinical symptoms of CS that overlap with much more prevalent conditions such as obesity, hypertension, and osteoporosis impede efficient case finding [4]. Different approaches have been tested in the past to improve diagnostic accuracy, such as face classification [15], diagnostic scores [16, 17], plasma steroid profiling [18], and measuring hair cortisol [19]. However, none of these approaches have been introduced into clinical practice. The standard screening still consists of a careful history, clinical examination, and the use of three standard screening tests: measuring cortisol in a 24-hour collection of urine, the late-night salivary cortisol, and the $1 \mathrm{mg}$ low dose dexamethasone suppression test [2]. Salivary cortisol has also been proposed for surveillance of medical therapy in recurrent or persistent disease [20,21].

\section{Main results and implications for clinical practice}

The main findings of our study are two-fold: first, a five-point diurnal saliva cortisol profile improved the diagnostic accuracy of saliva cortisol measurement as a screening tool. Secondly, although the diurnal profiles between different subtypes of CS vary statistically significantly, there is no clinically meaningful application in the differential diagnosis because of strong overlap and variability. The high number of subjects and the vigorous methodology give our study the adequate basis for a firm clinical recommendation. Although saliva sampling can be easily conducted by the patient in an outpatient setting, based on our findings, we do not recommend diurnal cortisol profiles as a standard diagnostic tool for CS or a subtyping tool.

Surprisingly, patients with pituitary CS retain a circadian rhythm of cortisol secretion, which is shifted upwards, while patients with adrenal CS are characterized by a more "rigid" cortisol secretion pattern with reduced fluctuations. This is in line with the results of a study using plasma cortisol levels in patients with adrenal CS and
Cushing's disease [22]. In patients with ectopic CS, rhythm is completely lost. In clinical practice, distinguishing between patients with pituitary and ectopic CS is a challenging task. The identification of microadenomas in pituitary MRI is far from perfect [23], the inferior petrosal sinus invasive sampling and other tests like the high-dose dexamethasone- suppression test or the CRH-test lack specificity [24]. In these cases, salivary cortisol profiles might give an additional hint towards one or the other diagnosis.

Based on adrenal incidentalomas, patients with autonomous cortisol secretion have a quite different profile compared to patients with adrenal CS. Circadian rhythm is mostly intact. In comparison to control subjects, their afternoon and evening salivary cortisol levels are elevated. The mode of treatment of patients with autonomous cortisol secretion is still a matter of debate. Our data might confirm the approach to treat these patients with chronotherapy using medical therapy in the evening to lower cortisol levels [25] taking into account the importance of a circadian rhythm $[26,27]$. Of note, large prospective studies are needed to analyze the benefits of such an approach.

Besides, salivary cortisol profiles might also be used to tailor medical therapy according to a more physiological cortisol rhythm, as measuring salivary cortisol - besides urinary free cortisol - in patients with medical therapy is associated with improved quality of life $[20,21]$.

\section{Limitations and strengths}

Despite its thorough study design, this study has a few limitations including the monocentric design. Also, due to a low prevalence, the number of patients with ectopic CS was low. This study also has several important strengths; we analyzed a relatively large group of well-examined patients with a high number of patients with pituitary and adrenal CS. In addition, all salivary cortisol measurements were performed in the same laboratory.

\section{Conclusion}

Salivary cortisol day-profiles differ significantly between patients with and without CS and between different subtypes. They can be used to improve diagnostic accuracy and prevent false positive or false negative screening results, but the value is limited. 


\section{Conflict of Interest}

The authors state that they have no conflict of interest.

\section{References}

[1] Newell-Price J, Bertagna X, Grossman AB et al. Cushing's syndrome. Lancet (London, England) 2006; 367: 1605-1617. doi:10.1016/ s0140-6736(06)68699-6

[2] Nieman LK, Biller BM, Findling JW et al. The diagnosis of Cushing's syndrome: An Endocrine Society Clinical Practice Guideline. J Clin Endocrinol Metab 2008; 93: 1526-1540. doi:10.1210/jc.2008-0125

[3] Reimondo G, Pia A, Bovio S et al. Laboratory differentiation of Cushing's syndrome. Clinica Chim Acta 2008; 388: 5-14. doi:10.1016/j.cca.2007.10.036

[4] Braun LT, Riester A, Osswald-Kopp A et al. Toward a diagnostic score in Cushing's syndrome. Front Endocrinol 2019; 10: 766. doi:10.3389/ fendo.2019.00766

[5] Danet-Lamasou M, Asselineau J, Perez P et al. Accuracy of repeated measurements of late-night salivary cortisol to screen for early-stage recurrence of Cushing's disease following pituitary surgery. Clin Endocrinol 2015; 82: 260-266. doi:10.1111/cen.12534

[6] Amlashi FG, Swearingen B, Faje AT et al. Accuracy of late-night salivary cortisol in evaluating postoperative remission and recurrence in Cushing's disease. J Clin Endocrinol Metab 2015; 100: 3770-3777. doi:10.1210/jc.2015-2107

[7] Trilck M, Flitsch J, Lüdecke DK et al. Salivary cortisol measurement-a reliable method for the diagnosis of Cushing's syndrome. Exp Clin Endocrinol Diabetes 2005; 113: 225-230. doi:10.1055/s-2005-837667

[8] Castro M, Elias PC, Quidute AR et al. Out-patient screening for Cushing's syndrome: The sensitivity of the combination of circadian rhythm and overnight dexamethasone suppression salivary cortisol tests. J Clin Endocrinol Metab 1999; 84: 878-882. doi:10.1210/ jcem.84.3.5521

[9] Laudat MH, Cerdas S, Fournier C et al. Salivary cortisol measurement: A practical approach to assess pituitary-adrenal function. J Clin Endocrinol Metab 1988; 66: 343-348. doi:10.1210/jcem-66-2-343

[10] Berr CM, Di Dalmazi G, Osswald A et al. Time to recovery of adrenal function after curative surgery for Cushing's syndrome depends on etiology. J Clin Endocrinol Metab 2015; 100: 1300-1308. doi:10.1210/ jc.2014-3632

[11] Berr CM, Stieg MR, Deutschbein T et al. Persistence of myopathy in Cushing's syndrome: Evaluation of the German Cushing's Registry. Eur J Endocrinol 2017; 176: 737-746. doi:10.1530/eje-16-0689

[12] Braun LT, Fazel ], Zopp S et al. The effect of biochemical remission on bone metabolism in Cushing's syndrome: A 2-year follow-up study. J Bone Miner Res 2020; 35: 1711-1717. doi:10.1002/jbmr.4033
[13] Fassnacht M, Arlt W, Bancos I et al. Management of adrenal incidentalomas: European Society of Endocrinology Clinical Practice Guideline in collaboration with the European Network for the Study of Adrenal Tumors. Eur J Endocrinol 2016; 175: G1-g34. doi:10.1530/ eje-16-0467

[14] Nieman LK. Cushing's syndrome: Update on signs, symptoms and biochemical screening. Eur J Endocrinol 2015; 173: M33-M38. doi:10.1530/eje-15-0464

[15] Kosilek RP, Schopohl J, Grunke M et al. Automatic face classification of Cushing's syndrome in women - a novel screening approach. Exp Clin Endocrinol Diabetes 2013; 121: 561-564. doi:10.1055/s-0033-1349124

[16] Nugent CA, Warner HR, Dunn JT et al. Probability theory in the diagnosis of Cushing's syndrome. J Clin Endocrinol Metab 1964; 24: 621-627. doi:10.1210/jcem-24-7-621

[17] Leon-Justel A, Madrazo-Atutxa A, Alvarez-Rios Al et al. A probabilistic model for Cushing's syndrome screening in at-risk populations: $A$ prospective multicenter study. J Clin Endocrinol Metab 2016; 101 3747-3754. doi:10.1210/jc.2016-1673

[18] Eisenhofer G, Masjkur ], Peitzsch M et al. Plasma steroid metabolome profiling for diagnosis and subtyping patients with Cushing syndrome. Clin Chem 2018; 64: 586-596. doi:10.1373/clinchem.2017.282582

[19] Savas M, Wester VL, de Rijke YB et al. Hair glucocorticoids as a biomarker for endogenous Cushing's syndrome: Validation in two independent cohorts. Neuroendocrinology 2019; 109: 171-178. doi:10.1159/000498886

[20] Newell-Price J, Pivonello R, Tabarin A et al. Use of late-night salivary cortisol to monitor response to medical treatment in Cushing's disease. Eur J Endocrinol 2020; 182: 207-217. doi:10.1530/eje-190695

[21] Yoshida K, Fukuoka H, Odake Y et al. Multiple salivary cortisol measurements are a useful tool to optimize metyrapone treatment in patients with Cushing's syndromes treatment: Case presentations. Front Endocrinol 2017; 8: 375. doi:10.3389/fendo.2017.00375

[22] Van Cauter E, Refetoff S. Evidence for two subtypes of Cushing's disease based on the analysis of episodic cortisol secretion. N Engl J Med 1985; 312: 1343-1349. doi:10.1056/nejm198505233122102

[23] Ikeda H, Abe T, Watanabe K. Usefulness of composite methioninepositron emission tomography/3.0-Tesla magnetic resonance imaging to detect the localization and extent of early-stage Cushing adenoma. J Neurosurg 2010; 112: 750-755. doi:10.3171/2009.7.jns09285

[24] Ritzel K, Beuschlein F, Berr C et al. ACTH after 15 min distinguishes between Cushing's disease and ectopic Cushing's syndrome: A proposal for a short and simple CRH test. Eur J Endocrinol 2015; 173 : 197-204. doi:10.1530/eje-14-0912

[25] Braun LT, Reincke M. What is the role of medical therapy in adrenaldependent Cushing's syndrome? Best Pract Res Clin Endocrinol Metab 2020; 34: 101376. doi:10.1016/j.beem.2020.101376

[26] Debono M, Harrison RF, Chadarevian R et al. Resetting the abnormal circadian cortisol rhythm in adrenal incidentaloma patients with mild autonomous cortisol secretion. J Clin Endocrinol Metab 2017; 102: 3461-3469. doi:10.1210/jc.2017-00823

[27] Minnetti M, Hasenmajer V, Pofi R et al. Fixing the broken clock in adrenal disorders: Focus on glucocorticoids and chronotherapy. J Endocrinol 2020; 246: R13-r31. doi:10.1530/joe-20-0066 